\title{
A Review of Coronary Vessel Segmentation Algorithms
}

\author{
Maryam Taghizadeh Dehkordii, ${ }^{1,2}$ Saeed Sadri ${ }^{1,2}$, Alimohamad Doosthoseini ${ }^{1}$ \\ ${ }^{1}$ Departments of Electrical and Engineering, Isfahan University of Technology, ${ }^{2}$ Medical Image and Signal Processing Research Center, \\ Isfahan University of Medical Sciences, Isfahan, Iran
}

\section{A B S T R A C T}

Coronary heart disease has been one of the main threats to human health. Coronary angiography is taken as the gold standard; for the assessment of coronary artery disease. However, sometimes, the images are difficult to visually interpret because of the crossing and overlapping of vessels in the angiogram. Vessel extraction from X-ray angiograms has been a challenging problem for several years. There are several problems in the extraction of vessels, including: weak contrast between the coronary arteries and the background, unknown and easily deformable shape of the vessel tree, and strong overlapping shadows of the bones. In this article we investigate the coronary vessel extraction and enhancement techniques, and present capabilities of the most important algorithms concerning coronary vessel segmentation.

Key words: Coronary vessel, vessel segmentation, X-ray angiography

\section{INTRODUCTION}

Correct assessment, especially accurate visualization and quantification of blood vessels reflected in X-ray angiograms or angiography images, plays a significant role in a number of clinical procedures. For various medical diagnostic tasks, it is necessary to measure the vessel width, reflectivity, tortuosity, and abnormal branching. For example, detecting the occurrence of vessels of a certain width may reveal the signs of stenosis. Grading of stenosis is of importance to diagnose the severity of vascular disease and to determine the treatment therapy ${ }^{[1,2]}$ Moreover, planning and performing neurosurgical procedures require an exact insight into blood vessels and their branches, which exhibit great variability. In planning, they provide information on where the blood is drawn and drained, to differentiate between the feeding and transgressing vessel. Even as transgressing vessels need to be preserved, the feeding ones are selectively closed through the artery in interventional neuroradiology, such as the brain arteriovenous malformation treatment. During surgery the vessels serve to provide landmarks and guidelines to the lesion. In short, accuracy in the navigation and localization of clinical procedures is determined by how minute and subtle the vascular information is. Although it is possible for medical experts to delineate vessels, manual delineation of the vasculature becomes tedious or even impossible when the number of vessels in an image is large or when a large number of images is acquired. ${ }^{[3]}$ Therefore, the development of automatic and accurate vessel-tree reconstruction from angiograms is highly desirable. However, it has proven to be a challenging task. The key fact is that vessels cannot be characterized uniformly. Thick vessels have more contrast to noise ratios, as compared to small narrow ones, due to the strong presence of blood and contrast agents in the vessels [Figure 1a]. Non-uniform illumination, as shown in Figure 1b, is one of the major sources of angiography image degradation,,${ }^{|3|}$ and is also a hindrance for accurate reconstruction, because it is likely to make an individual vessel break into several segments. We have divided the image processing methods used in coronary angiography into two main categories: (1) vessel enhancement (2) vessel segmentation. These categories are further divided into subcategories. According to, ${ }^{[4,5]}$ the current automatic computer-assisted procedures are still far from providing a precise spatial representation of the vessel tree.

\section{VESSEL ENHANCEMENT}

The literature on enhancing X-ray coronary angiographic images for visualization purposes is very limited. Although a number of algorithms have been proposed for angiographic image enhancement, the purpose of most algorithms is to improve the subsequent segmentation rather than visualization. These algorithms can hardly be adopted in clinical practice for improving the quality of visualization.

Algorithms based on specific noise models, for example, the quantum noise model, ${ }^{[6]}$ might also fail to work in practice, 
as image noise, that is, the undesirable appearance of mottled or grainy spots, which do not reflect the true tissue property, is a combination of various sources of noise, with different characteristics.

Attempting to increase the contrast of vascular structures by suppressing or removing the background structures, for example, the piecewise normalization ${ }^{[7]}$ and the rolling algorithm, ${ }^{[8]}$ is also of limited effect, as part of image noise, with the intensity value within the range of foreground structures, for example, vessels, will be enhanced as well. The step of removing the background might at the same time remove some detailed information in low-contrast angiographic images, which is very undesirable. To the best of the authors' knowledge, all angiographic acquisition systems available in the market use a certain technique to enhance the acquired images in real time, that is, during the actual acquisition procedure. Most of these enhancement techniques are based on the so-called unsharp masking technique and allow the operators to customize the degree of enhancement by using multiple gain levels (typically five). The unprocessed image is first blurred and subtracted from the original image, creating an edge image that only contains higher spatial frequency components of the original image. This edge image is further multiplied by a certain gain level and added to the original image, resulting in an edge enhanced image. ${ }^{[9]}$ Although the image edges are visually enhanced, the result is less optimal, as the image noise with a high spatial frequency will also be enhanced, which may introduce an undesirable appearance or influence the perception of the image details.

The other method used for the enhancement of angiography images is registration of algorithms. Image registration is a useful approach to correct artifacts. In this technique, the correspondence between pixels in mask and live images are calculated. Then a certain warping method is applied to the mask image. After subtracting the two images, the artifacts can be greatly eliminated. ${ }^{[10]}$ First, ${ }^{[11]}$ the mask image is decomposed into many sub-image blocks. With a template matching method, based on typical similarity measures, the rigid matching between the mask sub-image blocks and the live image can be achieved. Second, the control point pairs are selected with Harris corner detection, and are extracted in the original and transformed mask images. By using the multilevel B-spline as the mapping function, a global elastic registration is accomplished. Figure 2 gives the registration results. ${ }^{[11]}$

\section{VESSEL SEGMENTATION}

Many segmentation methods have been used to visualize blood vessel structures in the human body. These blood vessel segmentation methods may be classified as follows: Pattern recognition, model-based tracking and propagation, neural network, fuzzy, and artificial intelligence-based

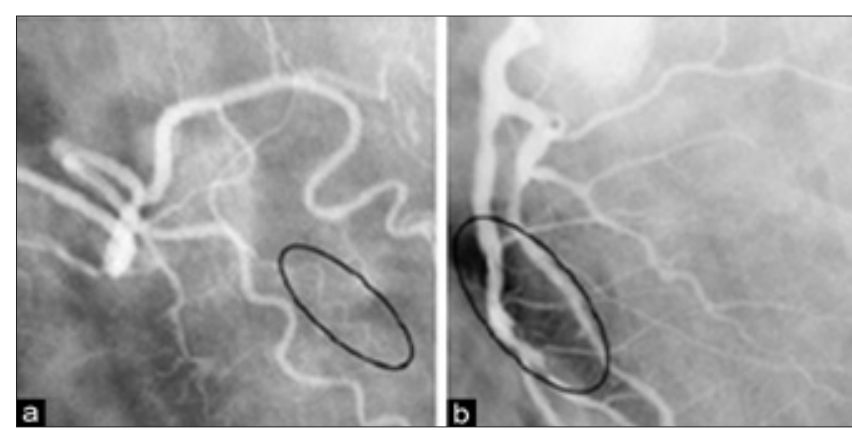

Figure I: Angiography images with typical hindrances for accurate vesseltree reconstruction such as (a) low-contrast vessels and (b) non-uniform illumination ${ }^{[5]}$

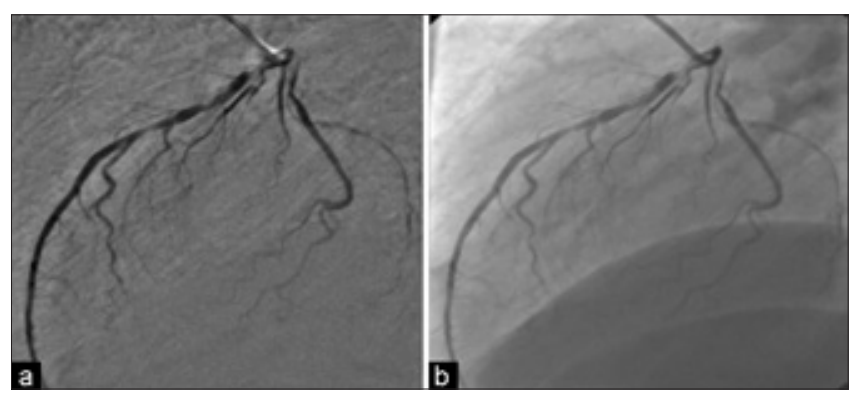

Figure 2: (a) The subtraction image after registration; (b) Original live image $^{[11]}$

methods. ${ }^{[12-20]}$ Artificial Intelligence-based approaches utilize knowledge to guide the segmentation process and delineate vessel structures. Different types of knowledge are employed in different systems from various sources. One knowledge source is the technique of acquisition of the properties of the image, such as cine-angiography, digital signature algorithm (DSA), computed tomography (CT), magnetic resonance imaging (MRI), and magnetic resonance angiography (MRA). Some applications utilize a general blood vessel model as a knowledge source. A statistical method is also presented for vessel segmentation. ${ }^{[21]}$

Poli and Valli ${ }^{[22]}$ proposed a computationally efficient algorithm based on a set of linear filters, obtained as linear combinations of properly shifted Gaussian kernels, sensitive to vessels of different orientation and radius. Another type of linear filter, the morphologically connected set filter, was utilized by Wilkinson and Westenberg, ${ }^{[23]}$ to capture filamentous structures. Together with a shape criterion that could distinguish filamentous structures from others, connected set filters could help to extract filamentous details without distortion. Similarly, Eiho and Qian ${ }^{[24]}$ and Zana and Klein ${ }^{[25]}$ used morphological operators such as erosion, dilation, and top-hat to enhance the shape of the artery and remove other points. These methods were unable to suppress sudden noise and edge noise and were less efficient in capillaries. Nonlinear anisotropic filtering was also applied for vessel enhancement. ${ }^{[26-29]}$ This method searched for the local 
orientation of a vessel to perform anisotropic smoothing without blurring its edge. While Krissian et al. ${ }^{[26]}$ performed a particular version of anisotropic diffusion, Orkisz et al. ${ }^{[29]}$ used a kind of filter bank called 'sticks', which could be seen as a set of directional structuring elements. Similar approaches were also proposed by Czerwinski et al., ${ }^{[30,31]}$ Kutka and Stier, ${ }^{[32]}$ Chen and Hale, ${ }^{[33]}$ and Du et al ${ }^{[34,35]}$ The last two references combined the outputs of directional operators, without an explicit extraction of the vessel local orientation.

The main disadvantage of the methods in this category is that they can hardly detect vessels in a wide range due to the fixed scale analysis. Although these algorithms can be extended to multiple scales by using sticks of variable length, the computation time would increase greatly. Hessian-based multiscale filtering has been proposed in a number of vessel enhancement approaches. ${ }^{[36-41]}$ One advantage of this approach, in this category, is that vessels in a wide range of diameters can be captured due to the multiscale analysis. In this method, an input image is first convolved with the derivatives of a Gaussian at multiple scales and then the Hessian matrix is analyzed at each pixel in the resulting image, to determine the local shape of the structures at that pixel. The ratio between the minimum and the maximum Hessian eigenvalues is small for linelike structures, but it must be high for blob-like ones. Krissian et al..$^{[42]}$ has specifically introduced several models of vessels and has used Hessian eigenvalues to define a set of candidate pixels, which can be the centerlines of the vessels. For each of these candidates, pre-defined, multiscale response functions have been computed, to determine the likelihood of the pixels corresponding to vessels of different scales (radii). The drawbacks of the Hessian-based approaches are that they are highly sensitive to noise, due to the second-order derivatives, and they tend to suppress junctions, as junctions are characterized similar to the blob-like structures. Junction suppression leads to the discontinuity of the vessel network, which is of course undesirable. To deal with this problem, Agam et al. ${ }^{[43]}$ proposed a filter model that is based on the correlation matrix of the regularized gradient vectors (first-order derivatives), to avoid the need for second-order derivatives. This model generated a good performance when dealing with thoracic CT images. However, when dealing with angiography images, which are noisier and suffer from non-uniform illumination, it shares the same limitations of Hessian-based filters in finding small and low-contrast vessels. The reason is that it is still using the Hessian eigenvalues to pre-select the vessel-candidate pixels at which the filter is applied. $\operatorname{In}^{[5]}$ a new framework proposed for the vessel enhancement filter, Utilizing the directional information present in an image. The proposed approach alleviates the calculation of the Hessian eigenvalues in a noisy environment. Specifically, the input image is first decomposed by a decimation-free directional filter bank
(DDFB) into a set of directional images, each of which contains line-like features in a narrow directional range. The directional decomposition has two advantages. One is, the noise in each directional image will be significantly reduced compared to that in the original one due to its omnidirectional nature. The other is, because a one-directional image contains only vessels with similar directions, this decomposition-filtering-recombination scheme also helps to preserve junctions.

The directional images are recombined to generate the output image with enhanced vessels, and the suppressed directional images are recombined to generate the output image with enhanced vessels, and the suppressed applied to enhance vessels in the respective directional images. Finally, the enhanced experimental results show that this approach is less noise-sensitive, can reveal the small vessel network, and avoid junction suppression.

Figure 3 gives the segmentation results ${ }^{[5]}$ and comparison with the Frangi and Shikata models. An edge detection method was also employed to extract edges that may be parallel to the opposing edges of the vessel segment. These methods use an adaptive threshold scheme to extract the vascular segments from medical images. Computation of this threshold value may not be satisfactory for all the qualities of the images.

On the other hand, ridge tracing tubular objects and several other approaches were used to track the vascularity in a medical image. An adaptive tracking method was also presented to extract the extended tracts of vascularity in the X-ray angiograms. ${ }^{[15,22]}$ Model-based approaches for explicit vessel structures were also applied to extract the vascularity in a medical image. One of these approaches used a deformable spline or the snake model to extract the vessel structures ${ }^{[12,23]}$ by deforming the spline or the snake to minimize some energy functions. Most of the present snake models could not provide a better capture range or evolution stop mechanism.

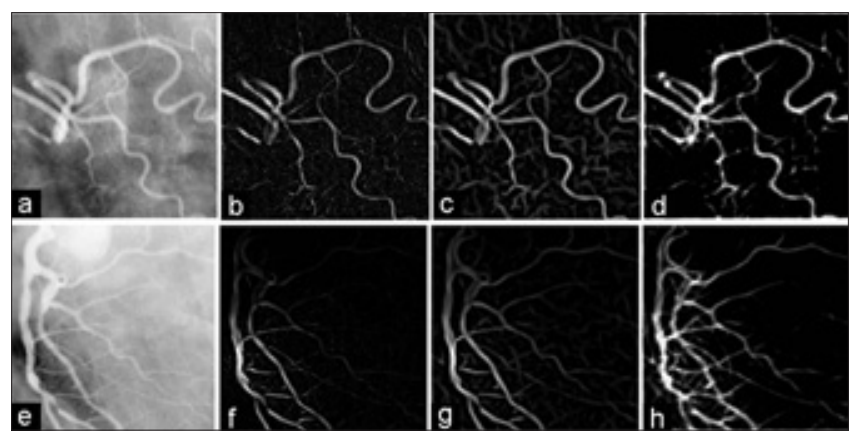

Figure 3: Qualitative results for two cardiac angiography images. (a and e) Original images, (b and $f$ ) enhanced images by Frangi method, $43.22 \mathrm{~s}$ each, (c and g) by Shikata method, 43.I I s, and ( $\mathrm{d}$ and $\mathrm{h}$ ) by the DFB-based approach, $49.08 \mathrm{~s}$. The Frangi and Shikata models fail to enhance small vessels accurately, but the DFB-based approach succeeds ${ }^{[5]}$ 
$\mathrm{A}^{[44]}$ new external force for active contours is presented, which solves both the problems. An extension of the gradient vector flow snake (GVF snake) method is presented. First, adaptive balloon force has been developed to increase both the GVF snake's capture range and convergence speed. Subsequently, a dynamic GVF force is introduced to provide an efficient evolution-stop mechanism [Figure 4].

Artificial intelligence-based approaches use a rule- and knowledge-based expert system, to segment coronary vessels from digital subtracted angiograms..$^{[4,18,45]}$

Automated systems and high processing throughput are needed in computationally intensive tasks, including visualization of the coronary blood flow and threedimensional reconstruction of the vascular structure from biplane medical images. ${ }^{[12,14-16,18,20]}$ The previous blood vessel segmentation algorithms are limited by at least one of the following drawbacks. The drawbacks are, applicability of the method for a limited number of morphologies, the need of user involvement to select the region of interest, and lack of adaptive capabilities, which results in poor quality of the segmentation, requiring a large computational effort for blood vessel segmentation. $\mathrm{A}^{[46]}$ model-based segmentation method is introduced for extracting blood vessel structures from poor quality coronary angiograms. This method extracts blood vessels in the angiograms by exploiting the spatial coherence existing in the image. Figure 5 gives the segmentation results. ${ }^{[46]}$

Table 1 presents the capabilities of some important algorithms mentioned earlier for coronary vessel segmentation. Coronary X-ray angiography is taken as the 'gold standard' for the assessment of coronary artery disease.

Thus, algorithms presented in Table 1 are specifically used for X-ray angiography. In this table, the comparison is based on different aspects. For instance as long as preprocessing is concerned, algorithms presented in the second and the third row need preprocessing. By comparing the results of these algorithms, the Truc ${ }^{[5]}$ can be seen by using multiscale analysis, together with directional filters, and an acceptable performance is obtained in the segmentation of coronary vessels.

\section{CONCLUSION}

In this article we have tried to cover both early and recent literature related to coronary vessel segmentation algorithms and techniques. Our aim was to introduce the
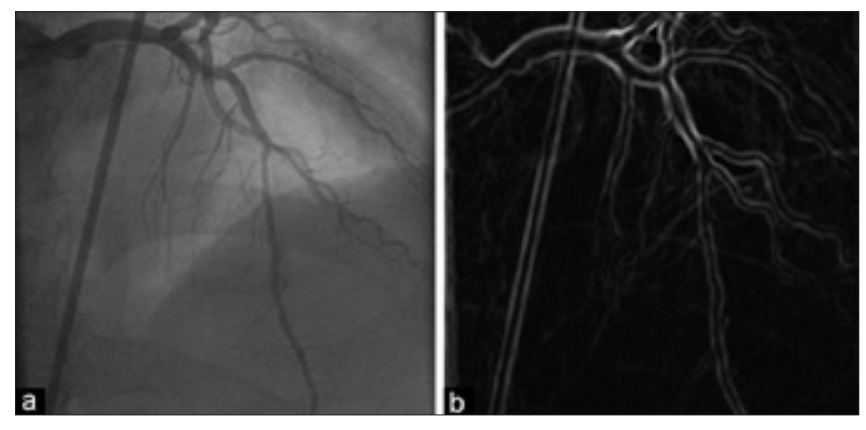

Figure 4: Normal X-ray coronary angiogram. (a) Input image, (b) improved GVF method ${ }^{[44]}$

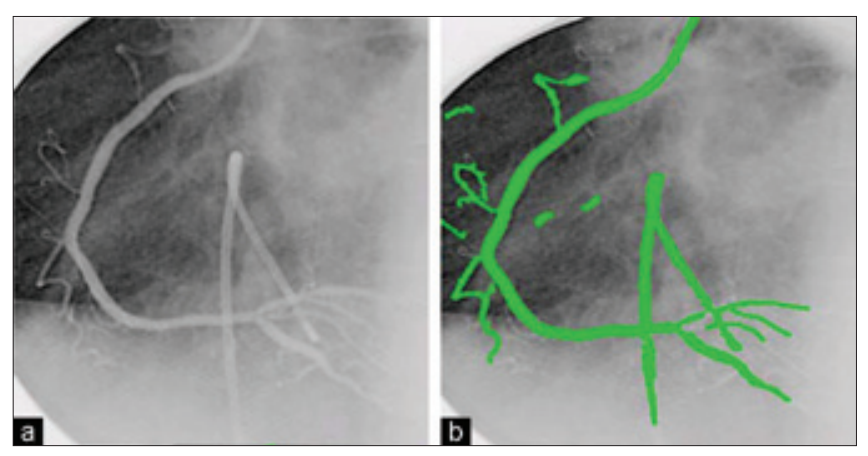

Figure 5: (a) A real image and (b) its segmentation result ${ }^{[46]}$

\begin{tabular}{|c|c|c|c|c|c|c|c|c|c|c|}
\hline Algorithm & Year & Classification & Input type & $\begin{array}{l}\text { Multi- } \\
\text { scale }\end{array}$ & $\begin{array}{c}\text { Pre- } \\
\text { processing }\end{array}$ & $\begin{array}{c}\text { A prior } \\
\text { knowledge }\end{array}$ & $\begin{array}{c}\text { User } \\
\text { interaction }\end{array}$ & Centerline & Junction & $\begin{array}{c}\text { Whole } \\
\text { tree }\end{array}$ \\
\hline Poli and Valli[i[22] & 1997 & PRA & XRA & $\checkmark$ & $\times$ & $\checkmark$ & $\checkmark$ & $\checkmark$ & $\checkmark$ & $\checkmark$ \\
\hline $\begin{array}{l}\text { Eiho and } \\
\text { Qian }^{[24]}\end{array}$ & 1997 & PRA & $\begin{array}{l}\text { Coronary } \\
\text { XRA }\end{array}$ & $x$ & $\checkmark$ & $\checkmark$ & $\checkmark$ & $\checkmark$ & $\checkmark$ & $\checkmark$ \\
\hline Klein et al. ${ }^{[12]}$ & 1997 & MBA & $\begin{array}{l}\text { Coronary } \\
\text { XRA }\end{array}$ & $\times$ & $\checkmark$ & $\checkmark$ & $\checkmark$ & $\checkmark$ & $x$ & $x$ \\
\hline $\begin{array}{l}\text { Kottke and } \\
\text { Sun }{ }^{[14]}\end{array}$ & 1990 & AIBA & $\begin{array}{l}\text { Coronary } \\
\text { XRA }\end{array}$ & $\times$ & $x$ & $\checkmark$ & $x$ & $x$ & $\checkmark$ & $\checkmark$ \\
\hline $\operatorname{Devi}^{[44]}$ & 2008 & MBA & $\begin{array}{l}\text { Coronary } \\
\text { XRA }\end{array}$ & $\times$ & $x$ & $\checkmark$ & $\checkmark$ & $\times$ & $\checkmark$ & $\checkmark$ \\
\hline Cemal Köse ${ }^{[46]}$ & 2008 & MBA & $\begin{array}{l}\text { Coronary } \\
\text { XRA }\end{array}$ & $x$ & $x$ & $\checkmark$ & $x$ & $x$ & $\checkmark$ & $\checkmark$ \\
\hline Truc $c^{[5]}$ & 2009 & PRA & $\begin{array}{l}\text { Coronary } \\
\text { XRA }\end{array}$ & $\times$ & $\checkmark$ & $\checkmark$ & $x$ & $\times$ & $\checkmark$ & $\checkmark$ \\
\hline
\end{tabular}

XRA: X-Ray Angiography, MBA: Model-based approach, PRA: Pattern Recognition Approaches, AlBA: Artificial Intelligence-Based Approaches 
current segmentation techniques. We intended to give the practitioner a framework for the existing research.

Accuracy of the segmentation process is crucial according to the nature of the work and leads to more precise and repeatable radiological diagnostic systems. Accuracy can be improved by incorporating a priori information on vessel anatomy and making use of a high level knowledge guide for the segmentation algorithm. Even though expert knowledge and guidance is essential in segmentation systems, the sheer volume of the medical image data requires more automatic segmentation systems, to reduce the work load.

\section{REFERENCES}

1. North American Symptomatic Carotid Endarterectomy Trial (NASCET) Steering Committee, North American symptomatic carotid endarterectomy trial. Stroke 22, pp. 711-20, 1991.

2. European Carotid Surgery Trialists Collaborative Group. Randomised trial of endarterectomy for recently symptomatic carotid stenosis: Final results of the MRC European Carotid Surgery (ECST). Lancet 351, pp. 1379-87, 1999.

3. E. M. Haacke, R. W. Brown, M. R. Thompson, and R. Venkatesan. Magnetic Resonance Imaging: Physical Principles and Sequence Design. New York: Wiley; 1999.

4. C. Kirbas, and F. Quek. A review of vessel extraction techniques and algorithms. ACM Comput Surv 36, pp. 81-121, 2004.

5. P. T. Truc, M. A. Khan, Y. K. Lee, S. Lee, and T.S. Kim. Vessel enhancement filter using directional filter bank. Comput Vis Image Underst 113, pp. 101-12, 2009.

6. T. Aach, U. Schiebel, and Spekowius G. Digital image acquisition and processing in medical X-ray imaging. J Electron Imaging 8, pp.7-22, 1999.

7. M. S. Kumar, W. Liyang, T. Ram, S. S. Jasjit. DSA image enhancement via multi-resolution motion correction for interventional procedures: A robust strategy. Proceedings of the fifth IASTED International Conference: Biomedical Engineering. Innsbruck: ACTA Press; 2007.

8. D.L. Wllson, and E. J. Kaplan. Linear and morphological digital image enhancement of peripheral angiography images. Proceedings of SPIE: Medical imaging IV: image processing. Newport Beach, 1990.

9. P. M. van der Zwet, and J. H. Reiber. The influence of image enhancement and reconstruction on quantitative coronary arteriography. Int J Card Imaging 11, pp. 211-21, 1995.

10. E. H. Meijering, K.J.Zuiderveld, and M. A. Viergever. Image registration for digital subtraction angiography. Int J Comput Vis 31, pp. 227-46, 1999.

11. M. Nejati, S. Sadri, and R. Amirfattahi. Point-based image registration in digital subtraction angiography using multilevel B-spline, Computer Vision and Image Understanding, submitted 2010.

12. A. K. Klein, and A. Amin. Quantities coronary angiography with deformable spline models. IEEE Trans Med Imag 16, pp. 468-82, 1997.

13. A. P. Dhawan, and L. Aratal. Segmentation of medical images through competitive learning. Comput Methods Programs Biomed 40, pp. 20315, 1993.

14. D. P. Kottke, and Ying Sun. Segmentation of coronary arteriogram by iterative ternary classification. IEEE Trans Biomed Eng 37, pp.778-85, 1990.

15. I. Liu, and Y. Sun. Recursive tracking of vascular networking angiograms based on the detection-deletion scheme. IEEE Trans Med Imaging 12, pp. 334-41, 1993.

16. F. K. Quek, and C. Kirbas. Vessel extraction in medical images by wave- propagation and trace-back. IEEE Trans Med Imaging 20, pp. 117-31, 2001.

17. M. Gudmundsson, E. A. El-Kwae, and M. R. Kabuka. Edge Detection in medical images using a genetic algorithm. IEEE Trans Med Imaging 17, pp. 469-74, 1998.

18. S. Kobashi, N. Kamiura, Y. Hata, F. Miyawaki. Fuzzy information granulation on blood vessel extraction from 3D, TOF MRA image. Int J Pattern Recognit Artif Intell 14, pp. 409-25, 2000.

19. S. Osher, and J. A. Sethian. Fronts propagating with curvature dependent speed: Algorithms based on Hamilton Jacobi formulation. J Comput Phys 79, pp.12-49, 1988.

20. T. Kayikcioglu, A. Ganal, M. Turhal, and C. Köse. A surface based method for detection of coronary boundaries in poor quality X-ray. Pattern Recognit Lett 23, pp. 783-802, 2002.

21. C. Köse. Fully automatic segmentation of coronary vessel structures in poor quality X-ray angiograms images. Springer: Lecture Notes in Computer Science, vol.: LNCS 4109, pp. 72-82, Aug 2006.

22. R. Poli, and G. Valli. An algorithm for real-time vessel enhancement and detection. Comput Meth Prog Biomed 52, pp. 1-22, 1996.

23. M. Wilkinson, and M. Westenberg. Shape preserving filament enhancement filtering. In: Proc. Int. Conf. on Medical Image Computing and Computer-Assisted Intervention, LNCS, vol. 2208, Utrecht, The Netherlands; pp. 770-7, 2001.

24. S. Eiho, and Y. Qian. Detection of coronary artery tree using morphological operator. In Proc. IEEE Comput. Cardiol, pp. 525-8, 1997.

25. F. Zana, and J.-C. Klein. Segmentation of vessel-like patterns using mathematical morphology and curvature evaluation. IEEE Trans Image Process 10, pp. 1010-9, 2001.

26. C. Yan, S. Hirano, and Y. Hata. Extraction of blood vessel in CT angiography image aided by fuzzy logic. In Proc. IEEE Int. Conf. Signal Processing, pp. 926-9, 2000.

27. K. Krissian, G. Malandain, and N. Ayache. Directional anisotropic diffusion applied to segmentation of vessels in 3D images. In Proc. Scale-Space Theory in Computer Vision, LNCS, pp. 345-8,1997.

28. K. Krissian. Flux-based anisotropic diffusion applied to enhancement of 3-D angiogram. IEEE Trans Med Imaging 21, pp.1440-2, 2002.

29. M. Orkisz, C. Bresson, I. Magnin, O. Champin, and P. Douek, Improved vessel visualization in MR angiography by nonlinear anisotropic filtering. Magn Reson Med 37, pp. 914-9, 1997.

30. R. Czerwinski, D. Jones, W. O’Brien, Jr. An approach to boundary detection in ultrasound imaging. In: Proc. IEEE Ultrasonics Symposium, pp. 951-5, 1993.

31. R. Czerwinski, D. Jones, and W. O’Brien Jr. Detection of lines and boundaries in speckle images-application to medical ultrasound. IEEE Trans Med Imaging 18, pp.126-36, 1999.

32. R. Kutka, and S. Stier. Extraction of line properties based on direction fields. IEEE Trans Med Imaging 15, pp. 51-8, 1996.

33. H. Chen, and J. Hale. An algorithm for MR angiography image enhancement. Magn Reson Med 33, pp. 534-40, 1995.

34. Y. P. Du, D. L. Parker, and W. L. Davis. Vessel enhancement filtering in three dimensional MR angiography. J Magn Reson Imaging 5, pp. 353-9, 1995.

35. Y. P. Du, and D. L. Parker. Vessel enhancement filtering in threedimensional MR angiograms using long-range signal correlation. J Magn Reson Imaging 7, pp. 447-50, 1997.

36. A. Frangi, W. Niessen, K. Vincken, and M. Viergever. Multiscale vessel enhancement filtering, in: Medical Image Computing ComputerAssisted Intervention. Lect Notes Comput Sci 1496, pp. 130-7, 1998.

37. A. Frangi, W. Niessen, R. Hoogeveen, T. van Walsum, and M. Viergever. Modelbased quantitation of 3-D magnetic resonance angiographic images. IEEE Trans Med Imaging 18, pp. 946-56, 1999.

38. A. Frangi, W. Niessen, P. Nederkoorn, J. Bakker, W. Mali, and M. Viergever. Quantitative analysis of vascular morphology from 3D MR 
angiograms: In vitro and in vivo results. Magn Reson Med 45, pp. 31122,2001

39. Y. Sato, S. Nakajima, N. Shiraga, H. Atsumi, S. Yoshida, T. Koller, et al. Three-dimensional multi-scale line filter for segmentation and visualization of curvilinear structures in medical images. Med Image Anal 2, pp. 143-68, 1998.

40. C. Lorenz, I.-C. Carlsen, T. Buzug, C. Fassnacht, and J. Weese. A multiscale line filter with automatic scale selection based on the Hessian matrix for medical image segmentation, In Proc. Scale-Space Theories in Computer Vision, LNCS, vol. 1252, pp. 152-63, 1997.

41. H. Shikata, E.A. Hoffman, and M. Sonka. Automated segmentation of pulmonary vascular tree from 3D CT images, In Proc. SPIE Int. Symp. Medical Imaging, vol. 5369, San Diego, CA, pp. 107-16, 2004.

42. K. Krissian, G. Malandain, N. Ayache, R. Vaillant, and Y. Trousset. Model-based detection of tubular structures in 3D images. Comput Vis Image Underst 80, pp.130-71, 2000.

43. G. Agam, S. G. Armato, and C. Wu. Vessel tree reconstruction in thoracic CT scans with application to nodule detection. IEEE Trans Med Imaging 24, pp. 486-99, 2005.

44. S.N. Devi, and N. Kumaravel. Comparison of active contour models for image segmentation in X-ray coronary angiogram images. J Med Eng Technol 32, pp. 408-17, 2008.

45. R. T. Ritchings, and A. C. Colhester. Detection of anomalies on carotid angiograms. Pattern Recognition Lett 4, pp. 367-74, 1986.

46. C. Köse, and C. İkibaș. Segmentation of coronary vessel structures in X-ray angiogram images by using spatial pattern matching method. In Proc. 23 ${ }^{\text {rd }}$ IEEE International Symposium on Computer and Information Sciences, pp. 1-6, 2008.

How to cite this article: Dehkordi MT, Sadri S, Doosthoseini A. A review of coronary vessel segmentation algorithms. J Med Sign Sens 2011;1:49-54

Source of Support: Nil, Conflict of Interest: None declared

\section{BIOGRAPHIES}

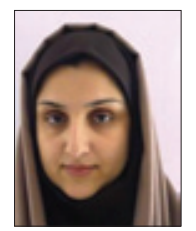

Maryam Taghizadeh Dehkordi received the B.Sc. degree in electrical engineering from the Isfahan University of Technology, Isfahan, Iran, in 2001. She received the M.Sc. degree in communication engineering in 2004, from the Isfahan University of Technology, Isfahan, Iran, Where she is currently a PhD student. Her main fields of interest include image processing.

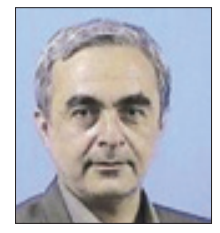

Saeed Sadri, Associate Professor in Electrical and Computers Engineering Department, Isfahan University of technology, Isfahan, Iran. He received the B.Sc. and the M.Sc. degree in electrical engineering from the University of Tehran, engineering from the Isfahan University of Technology, in 1997.

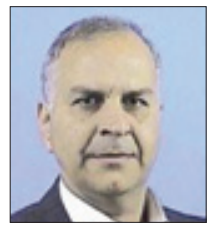

Alimohamad Doosthoseini, Associate Professor in Electrical and Computers Engineering Department, Isfahan University of technology, Isfahan, Iran. He received the $B$.Sc. degree in communication engineering from the Shiraz University, Shiraz, Iran, in 1975. He received the M.Sc. degree in communication engineering from the University of California, Berkeley, California, USA, in 1977. He received the $\mathrm{PhD}$ degree in communication engineering from the Stanford University, California, USA, in 1983.

Faculty of Engineering, Tehran, Iran, respectively in 1977 and 1979 . He received the $\mathrm{PhD}$ degree in communication

Announcement

Download iPhone, iPad application
A free application to browse and search the journal's content is now available for iPhone/iPad. The application provides "Table of Contents" of the latest issues, which are stored on the device for future offline browsing. Internet connection is required to access the back issues and search facility. The application is Compatible with iPhone, iPod touch, and iPad and Requires iOS 3.1 or later. The application can be downloaded from http://itunes.apple.com/us/app/medknow-journals/ id 458064375 ? $\mathrm{s}=18 \mathrm{mt}=8$. For suggestions and comments do write back to us. 